\title{
The Average Lower Connectivity of Graphs
}

\author{
Ersin Aslan \\ Turgutlu Vocational Training School, Celal Bayar University, Turgutlu, Manisa 45400, Turkey \\ Correspondence should be addressed to Ersin Aslan; ersin.aslan@cbu.edu.tr \\ Received 14 August 2013; Revised 8 January 2014; Accepted 9 January 2014; Published 12 February 2014 \\ Academic Editor: Bo-Qing Dong \\ Copyright (c) 2014 Ersin Aslan. This is an open access article distributed under the Creative Commons Attribution License, which \\ permits unrestricted use, distribution, and reproduction in any medium, provided the original work is properly cited. \\ For a vertex $v$ of a graph $G$, the lower connectivity, denoted by $s_{v}(G)$, is the smallest number of vertices that contains $v$ and those \\ vertices whose deletion from $G$ produces a disconnected or a trivial graph. The average lower connectivity denoted by $\kappa_{\text {av }}(G)$ is \\ the value $\left(\sum_{v \in V(G)} s_{v}(G)\right) /|V(G)|$. It is shown that this parameter can be used to measure the vulnerability of networks. This paper \\ contains results on bounds for the average lower connectivity and obtains the average lower connectivity of some graphs.
}

\section{Introduction}

In a communication network, the vulnerability parameters measure the resistance of the network to disruption of operation after the failure of certain stations or communication links. The best known and most useful measures of how well a graph is connected is the connectivity, defined to be the minimum number of vertices in a set whose deletion results in a disconnected or trivial graph. As the connectivity is the worst-case measure, it does not always reflect what happens throughout the graph. Recent interest in the vulnerability and reliability of networks (communication, computer, and transportation) has given rise to a host of other measures, some of which are more global in nature; see, for example, $[1,2]$.

Let $G$ be a finite simple graph with vertex set $V(G)$ and edge set $E(G)$. In the graph $G, n$ denotes the number of vertices. The minimum degree of a graph $G$ is denoted by $\delta(G)$. A subset $S \subset V(G)$ of vertices is a dominating set if every vertex in $V(G)-S$ is adjacent to at least one vertex of $S$. The domination number $\gamma(G)$ is the minimum cardinality of a dominating set. A subset $S$ of $V(G)$ is called an independent set of $G$ if no two vertices of $S$ are adjacent to $G$. An independent set $S$ is maximum if $G$ has no independent set $S^{\prime}$ with $\left|S^{\prime}\right|>|S|$. The independence number of $G, \alpha(G)$, is the number of vertices in a maximum independent set of $G$.

Henning [3] introduced the concept of average independence and average domination. For a vertex $v$ of a graph $G$, the lower independence number, denoted by $i_{v}(G)$, is the minimum cardinality of a maximal independent set of $G$ that contains $v$, and the lower domination number, denoted by $\gamma_{v}(G)$, is the minimum cardinality of a dominating set of $G$ that contains $v$. The average lower independence number of $G$, denoted by $i_{a v}(G)$, is the value $\left(\sum_{v \in V(G)} i_{v}(G)\right) /|V(G)|$ and the average lower domination number of $G$, denoted by $\gamma_{a v}(G)$, is the value $\left(\sum_{v \in V(G)} \gamma_{v}(G)\right) /|V(G)|$. Since $\gamma_{v}(G) \leq i_{v}(G)$ holds for every vertex $v$, we have $\gamma_{a v}(G) \leq i_{a v}(G)$ for any graph G. Also, it is clear that $i(G)=\min \left\{i_{v}(G) \mid v \in V(G)\right\}$ and $\gamma(G)=\min \left\{\gamma_{v}(G) \mid v \in V(G)\right\}$ so $\gamma(G) \leq \gamma_{a v}(G)$ and $i(G) \leq i_{a v}(G)$.

The $(u, v)$-connectivity of $G$, denoted by $\kappa_{G}(u, v)$, is defined to be the maximum value of $k$ for which $u$ and $v$ are $k$-connected. It is a well-known fact that the connectivity $\kappa(G)$ equals $\min \left\{\kappa_{G}(u, v) u, v \in V(G)\right\}$.

In 2002, Beineke et al. [4] introduced a parameter to give a more refined measure of the global "amount" of connectivity. If the order of $G$ is $n$, then the average connectivity of $G$, denoted by $\bar{\kappa}(G)$, is defined to be $\bar{\kappa}(G)=\left(\sum_{u, v} \kappa_{G}(u, v)\right) /\left(\begin{array}{l}n \\ 2\end{array}\right)$. The expression $\sum_{u, v} \kappa_{G}(u, v)$ is sometimes referred to as the total connectivity of $G$. Clearly, for any graph $G, \bar{\kappa}(G) \geq \kappa(G)$.

There are a lot of researches on the connectivity of a graph [5]. Many works provide sufficient conditions for a graph to be maximally connected [6-8]. The average connectivity has been extensively studied $[4,9]$.

\section{The Average Lower Connectivity of a Graph}

We introduce a new vulnerability parameter, the average lower connectivity. For a vertex $v$ of a graph $G$, the lower 


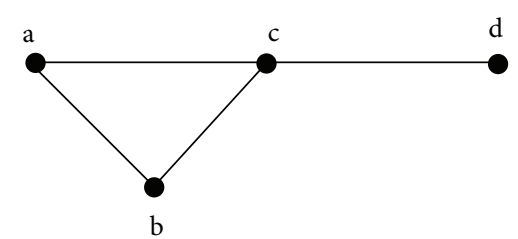

FIGURE 1: 3-cycle G: with one additional vertex and edge.

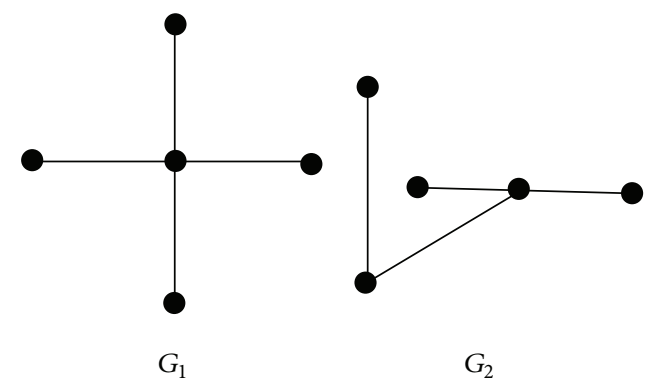

(a)

(b)

FIGURE 2: The graphs $G_{1}$ and $G_{2}$.

connectivity, denoted by $s_{v}(G)$, is the smallest number of vertices that contains $v$ and those vertices whose deletion from $G$ produces a disconnected or a trivial graph. We observe that

(i) $1 \leq s_{v}(G) \leq n-1$;

(ii) $s_{v}(G)=1$ if and only if $v$ is a cut vertex;

(iii) $s_{v}(G)=n-1$ if and only if $G-v$ is complete.

The average lower connectivity denoted by $\kappa_{a v}(G)$ is the value $\left(\sum_{v \in V(G)} s_{v}(G)\right) / n$, where $n$ will denote the number of vertices in graph $G$ and $\sum_{v \in V(G)} s_{v}(G)$ will denote the sum over all vertices of $G$. For any graph $G, \kappa(G)=\min \left\{s_{v}(G) \mid v \in V(G)\right\}$ so $\kappa_{\mathrm{av}}(G) \geq \kappa(G)$. We also observe that

(i) $\kappa_{\mathrm{av}}(G) \leq n-1$;

(ii) $\kappa_{\text {av }}(G)=n-1$ if and only if $G$ is complete.

Example 1. Let the graph $G$ be 3-cycle with one additional vertex and edge, as shown in Figure 1. It is easy to see that $s_{a}(G)=2, s_{b}(G)=2, s_{c}(G)=1$, and $s_{d}(G)=3$ and we have $\kappa_{a v}(G)=(2+2+1+3) / 4=2$.

Let $G_{1}$ and $G_{2}$ be graphs. Now one can ask the following question: is the average lower connectivity a suitable parameter? In other words, does the average lower connectivity distinguish between $G_{1}$ and $G_{2}$ ?

For example, consider the graphs in Figure 2.

It can be easily seen that the connectivity and average connectivity of these graphs are equal:

$$
\kappa\left(G_{1}\right)=\kappa\left(G_{2}\right)=\bar{\kappa}\left(G_{1}\right)=\bar{\kappa}\left(G_{2}\right)=1 .
$$

On the other hand, the average lower connectivity of $G_{1}$ and $G_{2}$ is different:

$$
\begin{aligned}
& \kappa_{\text {av }}\left(G_{1}\right)=1,8, \\
& \kappa_{\text {av }}\left(G_{2}\right)=1,6 .
\end{aligned}
$$

Thus, the average lower connectivity is a better parameter than the connectivity and average connectivity to distinguish these two graphs. The average parameters have been found to be more useful in some circumstances than the corresponding measures based on worst-case situations.

Theorem 2. Let $\mathrm{G}$ be a connected graph. Then,

$$
\kappa_{a v}(G)<\kappa(G)+2 .
$$

Proof. For every vertex of $G, s_{v}(G) \leq \kappa(G)+2$. For at least one vertex $v, s_{v}(G)=\kappa(G)$. Hence, the inequality is strict. Then,

$$
\kappa_{\mathrm{av}}(G)<\kappa(G)+2 .
$$

The proof is completed.

Theorem 3. Let $G$ be a connected graph. Then,

$$
\kappa_{a v}(G) \leq \kappa_{a v}(G+e) .
$$

Proof. It is easy to see that $s_{v}(G) \leq s_{v}(G+e)$. Therefore,

$$
\frac{\sum_{v \in V(G)} s_{v}(G)}{n} \leq \frac{\sum_{v \in V(G)} s_{v}(G+e)}{n} .
$$

So, we have

$$
\kappa_{\mathrm{av}}(G) \leq \kappa_{\mathrm{av}}(G+e) .
$$

The proof is completed.

Theorem 4. Let $G$ be a k-connected and k-regular graph. Then,

$$
\kappa_{a v}(G)=k .
$$

Proof. The cardinality of $s_{v}(G)$-sets is always the same for every vertex of any graph $G$ and equals $k$. Then, we have

$$
\kappa_{\mathrm{av}}(G)=\frac{\sum_{v \in V(G)} s_{v}(G)}{n}=\frac{n \cdot k}{n}=k .
$$

This means that the proof is completed.

It is obvious that we can give the following equality for the average lower connectivity of the cycle $C_{n}$.

(i) The average lower connectivity of the cycle $C_{n}$ is 2.

Theorem 5. Let $G$ be a connected graph. Then,

$$
\kappa_{a v}(G) \leq \delta(G)+2 .
$$

Proof. For every vertex of $G, s_{v}(G) \leq \delta(G)+2$. Thus,

$$
\begin{aligned}
\kappa_{\mathrm{av}}(G)= & \frac{\sum_{v \in V(G)} s_{v}(G)}{n} \leq \frac{n \cdot(\delta(G)+2)}{n}, \\
& \kappa_{\mathrm{av}}(G) \leq \delta(G)+2 .
\end{aligned}
$$

The proof is completed. 


\section{Average Lower Connectivity of Several Specific Graphs}

In this section, we determine the average lower connectivity of several special graphs.

Theorem 6. Let $T$ be a tree with order $n$. If $T$ has $k$ vertices with degree 1, then

$$
\kappa_{a v}(T)=\frac{n+k}{n} .
$$

Proof. Assume that $T$ has $k$ vertices with degree 1 and $n-k$ vertices with degree at least 2 . Let vertices set of $T$ be $V(T)=$ $V\left(G_{1}\right) \cup V\left(G_{2}\right)$ where in $V\left(G_{1}\right)$ the set contains $k$ vertices with degree 1; in $V\left(G_{2}\right)$ the set contains $n-k$ vertices with degree at least 2. If $v \in V\left(G_{1}\right)$, then $s_{v}\left(G_{1}\right)=2$. We have to repeat this process for $k$ vertices with degree 1 . If $v \in V\left(G_{2}\right)$, then $s_{v}\left(G_{2}\right)=1$. We have to repeat this process for $n-k$ vertices with degree at least 2 . Thus, we have

$$
\kappa_{\mathrm{av}}(T)=\frac{\sum_{v \in V(T)} s_{v}(T)}{|V(T)|}=\frac{k \cdot 2+(n-k) \cdot 1}{n}=\frac{n+k}{n} .
$$

The proof is completed.

Corollary 7. The average lower connectivity of

(a) the path $P_{n}$ is $(n+2) / n$;

(b) the star $K_{1, n-1}$ is $(2 n-1) / n$;

(c) the comet $C_{t, r}$ is $(2 r+t+1) /(t+r)$.

Theorem 8. Let $K_{r, s}$ be a complete bipartite graph. Then

$$
\kappa_{a v}\left(K_{r, s}\right)= \begin{cases}\frac{s^{2}+s r+r}{r+s}, & \text { if } s<r ; \\ r, & \text { if } r=s .\end{cases}
$$

Proof. Let the partite sets of $K_{r, s}$ be $R$ and $S$ with $|R|=r$ and $|S|=s$. We distinguish two cases.

Case 1. If $r=s$, then by Theorem 4 we have $\kappa_{\mathrm{av}}\left(K_{r, s}\right)=r$.

Case $2(r<s)$. For $x \in R$, a minimum disconnecting set of $G$ that contains $x$ must be $S \cup\{x\}$, so $s_{x}(G)=s+1$. On the other hand, for $y \in S$, a minimum disconnecting set of $G$ that contains $y$ must be $R$, so $s_{y}(G)=r$. Elementary computation yields the result.

Definition 9. The wheel graph with $n-1$ spokes, $W_{n}$, is the graph that consists of an $(n-1)$-cycle and one additional vertex, say $u$, that is adjacent to all the vertices of the cycle. In Figure 3, we display $W_{7}$.

Theorem 10. Let $W_{n}$ be a wheel graph. Then,

$$
\kappa_{a v}\left(W_{n}\right)=3 .
$$

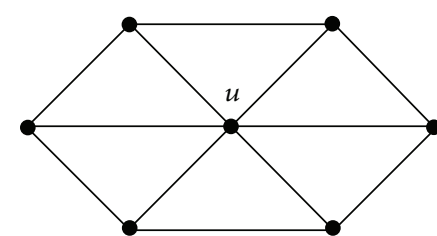

Figure 3: The wheel graph $W_{7}$.

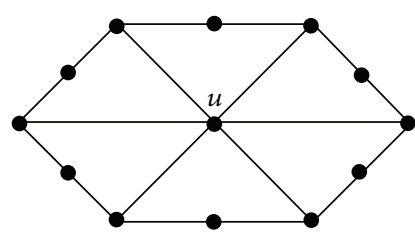

FIgURE 4: The gear graph $G_{6}$

Proof. The wheel graph $W_{n}$ has $n$ vertices. The cardinality of $s_{v}(G)$-sets is always the same for every vertex of any $W_{n}$ and equals 3 . Then, we have

$$
\kappa_{\mathrm{av}}\left(W_{n}\right)=\frac{\sum_{v \in V\left(W_{n}\right)} s_{v}\left(W_{n}\right)}{n}=\frac{n \cdot 3}{n}=3 .
$$

This means that the proof is completed.

Definition 11. The gear graph is a wheel graph with a vertex added between each pair adjacent to graph vertices of the outer cycle. The gear graph $G_{r}$ has $2 r+1$ vertices and $3 r$ edges. In Figure 4 we display $G_{6}$.

Theorem 12. Let $G_{r}$ be a gear graph. Then,

$$
\kappa_{a v}\left(G_{r}\right)=\frac{5 r+3}{2 r+1} .
$$

Proof. Let vertices set of $G_{r}$ be $V\left(G_{r}\right)=\left(H_{1}\right) \cup V\left(H_{2}\right) \cup V\left(H_{3}\right)$ where in $V\left(H_{1}\right)$ the set contains 1 vertex with degree $n, V\left(H_{2}\right)$ the set contains $n$ vertices with degree 2 , and $V\left(H_{3}\right)$ the set contains $n$ vertices with degree 3.If $v \notin V\left(H_{3}\right)$, then $s_{v}(G)=3$. If $v \in V\left(H_{3}\right)$, then $s_{v}\left(G_{3}\right)=2$. Therefore,

$$
\begin{aligned}
\kappa_{\mathrm{av}}\left(G_{r}\right) & =\frac{\sum_{v \in V\left(G_{r}\right)} s_{v}\left(G_{r}\right)}{2 r+1} \\
& =\frac{(r+1) \cdot 3+2 \cdot r}{2 r+1}=\frac{5 r+3}{2 r+1} .
\end{aligned}
$$

The proof is completed.

Now we give the definition of Cartesian product.

Definition 13. The Cartesian product $G_{1} \times G_{2}$ of graphs $G_{1}$ and $G_{2}$ has $V\left(G_{1}\right) \times V\left(G_{2}\right)$ as its vertex set and $\left(u_{1}, u_{2}\right)$ is adjacent to $\left(v_{1}, v_{2}\right)$ if either $u_{1}=v_{1}$ and $u_{2}$ is adjacent to $v_{2}$ or $u_{2}=v_{2}$ and $u_{1}$ is adjacent to $v_{1}$.

Connectivity of graph products has already been studied by different authors. In [10] it is proved that $\kappa\left(G_{1} \times G_{2}\right) \geq$ $\kappa\left(G_{1}\right)+\kappa\left(G_{2}\right)$. 
Theorem 14. Let $G_{1}$ and $G_{2}$ be two connected graphs; then

$$
\kappa_{a v}\left(G_{1} \times G_{2}\right) \geq \kappa\left(G_{1} \times G_{2}\right) .
$$

Proof. We know $\kappa_{\mathrm{av}}(G) \geq \kappa(G)$. Therefore, we get

$$
\kappa_{\mathrm{av}}\left(G_{1} \times G_{2}\right) \geq \kappa\left(G_{1} \times G_{2}\right) .
$$

The proof is completed.

Proposition 15. For positive integer $m \geq 3$,

(i) $\kappa_{a v}\left(K_{2} \times P_{m}\right)=2$;

(ii) $\kappa_{a v}\left(K_{2} \times C_{m}\right)=3$.

Proposition 16. Let $r \geq 3$ and $t \geq 3$ be positive integers. Then

(i) $\kappa_{a v}\left(P_{r} \times P_{t}\right)= \begin{cases}\frac{23}{9}, & \text { if } r=3, t=3 ; \\ 3-\frac{2}{t}, & \text { if } r=3, t \geq 4 ; \\ 3-\frac{8}{r \cdot t}, & \text { if } r \geq 4, t \geq 4\end{cases}$

(ii) $\kappa_{a v}\left(P_{r} \times C_{t}\right)= \begin{cases}3, & \text { if } r=3,4, t \geq 3 ; \\ 4-\frac{4}{r}, & \text { if } r \geq 5, t \geq 4 .\end{cases}$

(iii) $\kappa_{a v}\left(C_{r} \times C_{t}\right)=4$.

\section{Conflict of Interests}

The author declares that there is noconflict of interests regarding the publication of this paper.

\section{Acknowledgment}

The author wishes to thank Bo-Qing Dong for his helpful suggestions and corrections.

\section{References}

[1] K. S. Bagga, L. W. Beineke, R. E. Pippert, and M. J. Lipman, "A classification scheme for vulnerability and reliability parameters of graphs," Mathematical and Computer Modelling, vol. 17, no. 11, pp. 13-16, 1993.

[2] O. R. Oellermann, "Connectivity and edge-connectivity in graphs: a survey," Congressus Numerantium, vol. 116, pp. 231252, 1996.

[3] M. A. Henning, "Trees with equal average domination and independent domination numbers," Ars Combinatoria, vol. 71, pp. 305-318, 2004.

[4] L. W. Beineke, O. R. Oellermann, and R. E. Pippert, "The average connectivity of a graph," Discrete Mathematics, vol. 252, no. 1-3, pp. 31-45, 2002.

[5] A. Hellwig and L. Volkmann, "Maximally edge-connected and vertex-connected graphs and digraphs: a survey," Discrete Mathematics, vol. 308, no. 15, pp. 3265-3296, 2008.

[6] Y.-C. Chen, "Super connectivity of $k$-regular interconnection networks," Applied Mathematics and Computation, vol. 217, no. 21, pp. 8489-8494, 2011.
[7] J. Fàbrega and M. A. Fiol, "Maximally connected digraphs," Journal of Graph Theory, vol. 13, no. 6, pp. 657-668, 1989.

[8] T. Soneoka, H. Nakada, M. Imase, and C. Peyrat, "Sufficient conditions for maximally connected dense graphs," Discrete Mathematics, vol. 63, no. 1, pp. 53-66, 1987.

[9] P. Dankelmann and O. R. Oellermann, "Bounds on the average connectivity of a graph," Discrete Applied Mathematics, vol. 129, no. 2-3, pp. 305-318, 2003.

[10] W.-S. Chiue and B.-S. Shieh, "On connectivity of the Cartesian product of two graphs," Applied Mathematics and Computation, vol. 102, no. 2-3, pp. 129-137, 1999. 


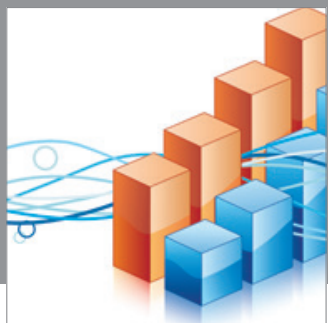

Advances in

Operations Research

mansans

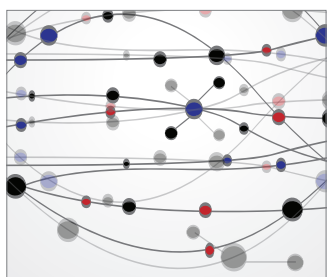

The Scientific World Journal
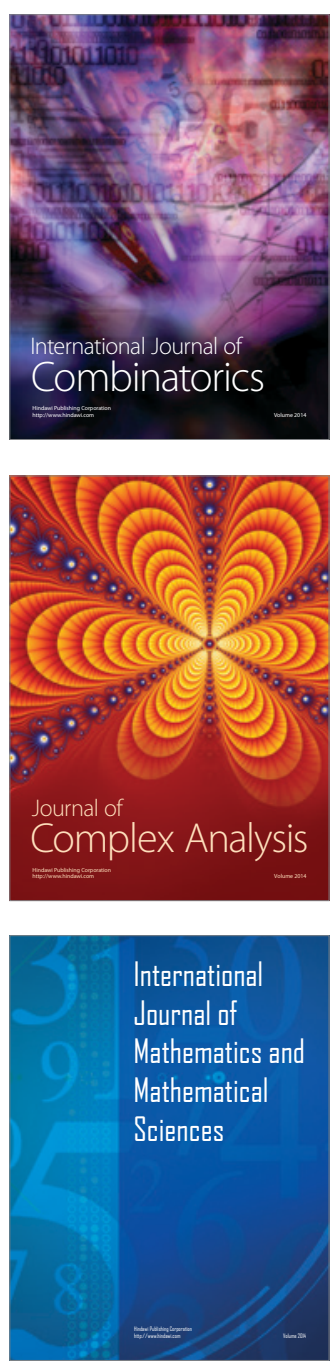
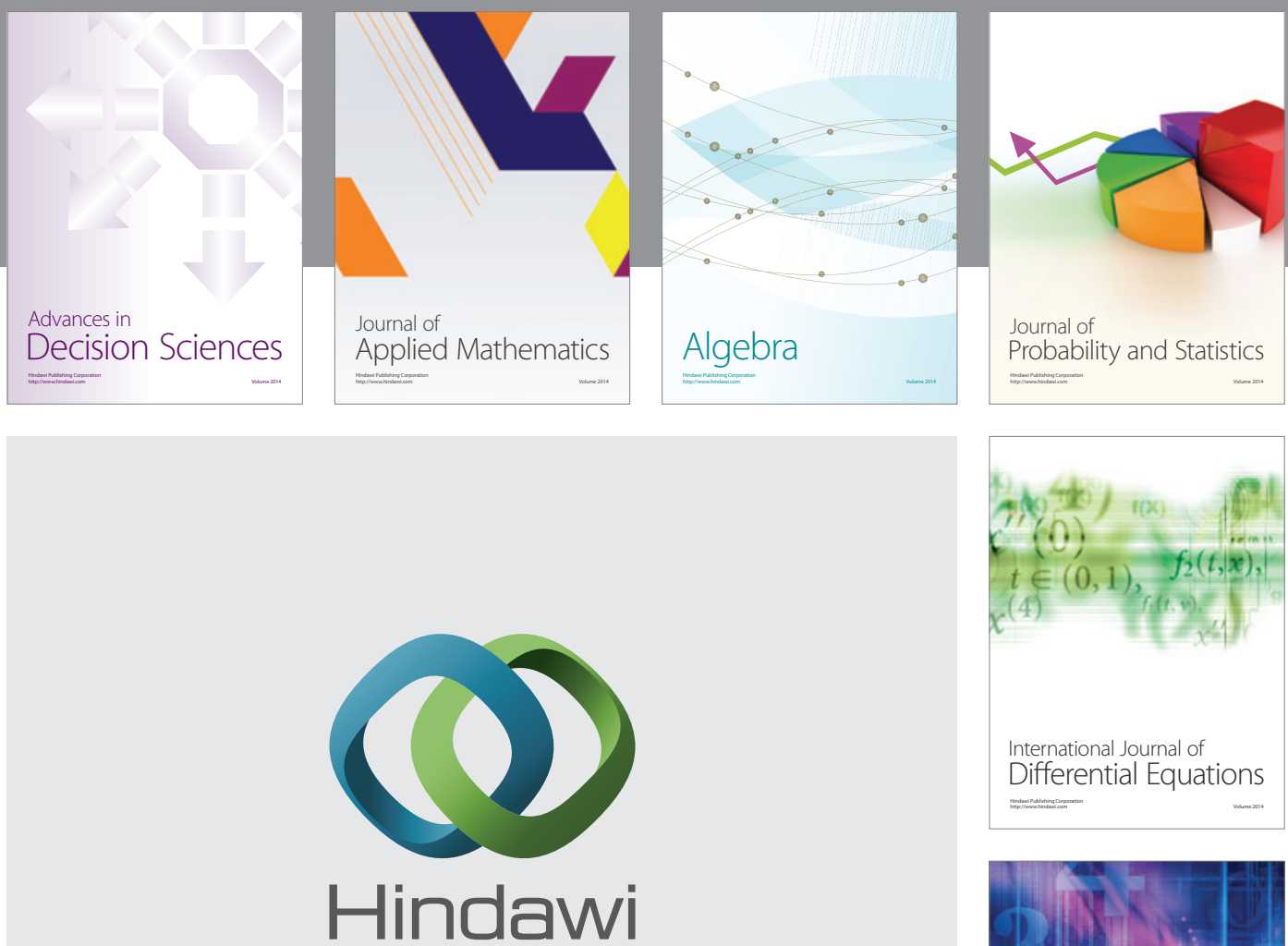

Submit your manuscripts at http://www.hindawi.com
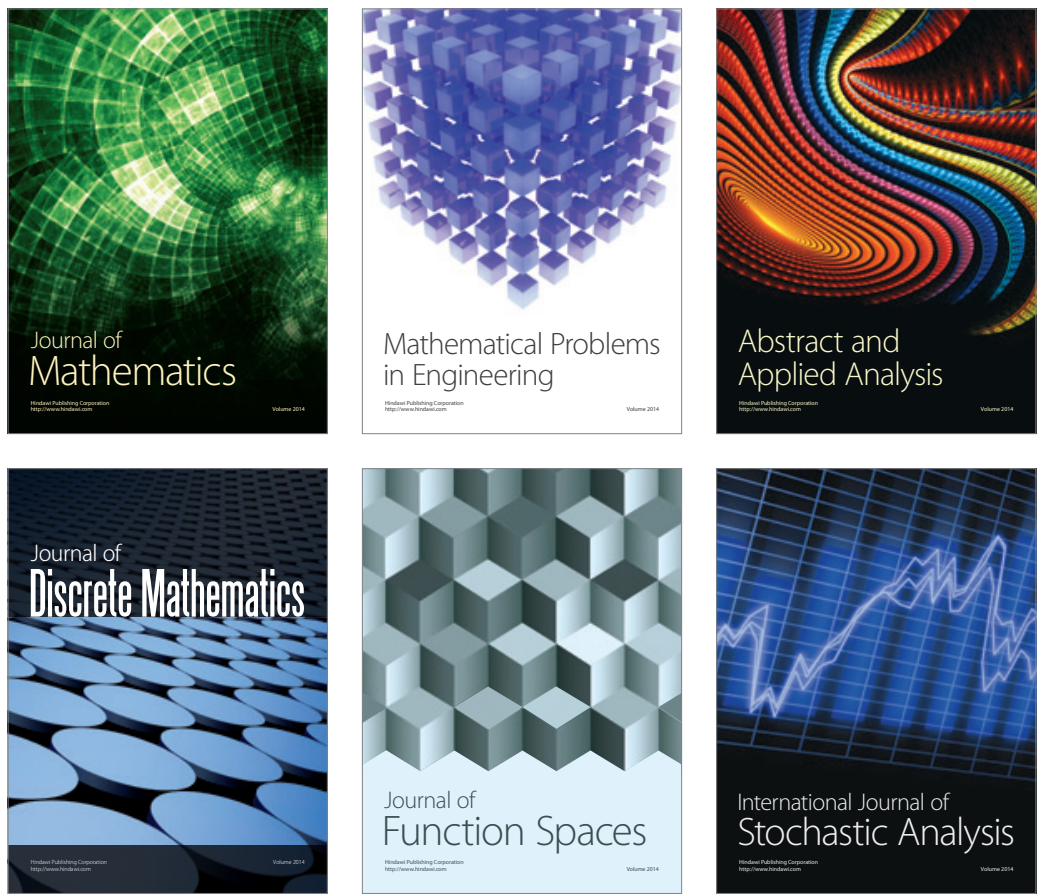

Journal of

Function Spaces

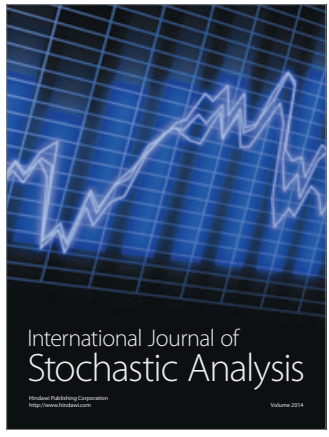

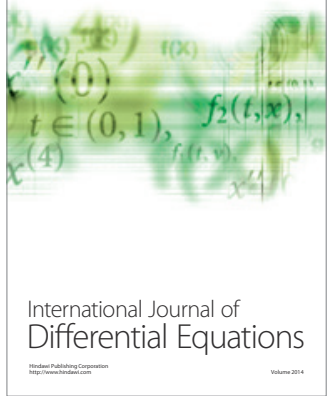
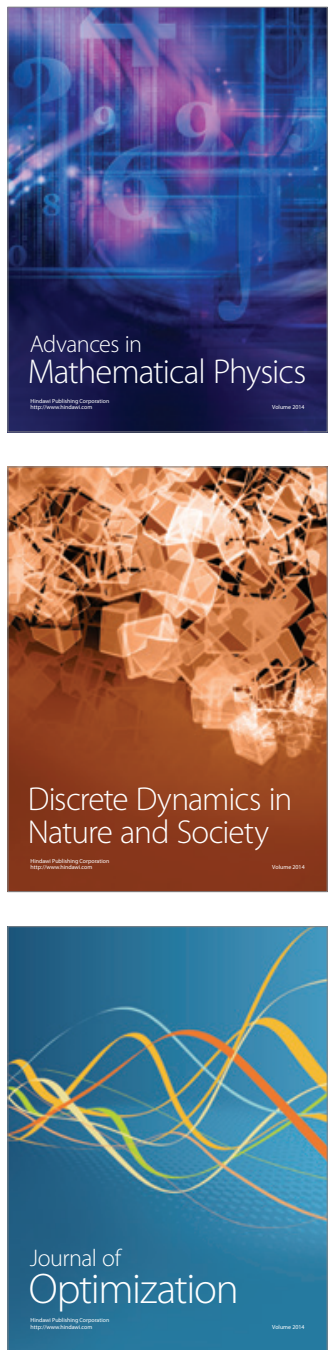